\section{Early relapse after autologous transplant for myeloma is associated with poor survival regardless of cytogenetic risk}

Multiple Myeloma (MM) management and survival have dramatically improved in the last decade, notably with triple combinations including one immunomodulatory drug (IMiD) and one proteasome inhibitor (PI) for induction treatment, and high dose therapy (HDT) plus autologous stem cell transplantation (ACST) in younger patients. However, while some patients experience long remission and even cure for rare cases, prognosis is still poor for high-risk MM patients. Early relapse (ER) after treatment has been recognized as an independent risk factor of resistance to rescue treatments and shorter overall survival (OS). ${ }^{1,2}$ Heterogeneity in treatment response duration may be influenced by several patient or disease related features such as frailty, age, comorbidity, clinical stage and cytogenetic abnormalities. Among the highrisk chromosomal changes described in MM, those established and on which there is general consensus are $\operatorname{del}(17 \mathrm{p})$ and $t(4 ; 14)$. These abnormalities impact nega- tively both, progression free survival (PFS) and OS, and affect respectively around $8 \%$ and $15 \%$ of newly diagnosed multiple myeloma (NDMM) patients. ${ }^{3,4}$

Given the current availability of particularly effective salvage therapies, ${ }^{5}$ we wondered if an ER after first line intensive treatment is still associated with poor OS in both high-risk and standard-risk patients. To address this issue, we retrospectively analyzed clinical and cytogenetic data of a large and homogeneous cohort of NDMM patients treated by intensive chemotherapy followed by ACST, and consolidation.

The Toulouse Ethics Committee approved the study and written informed consent was obtained for all patients included. Clinical data were obtained from 2,627 patients followed $>18$ months or having relapsed, and for whom diagnosis of $\mathrm{MM}$ was established between April 1, 2004 and August 17, 2018. Patients who had primary refractory disease were not included. All patients received an intensive approach with either a doubletbased induction $(38 \%)$ including PI or a triplet-based $(62 \%)$ including a PI and an IMiD, followed by ASCT and consolidation by same regimen as induction. Twelve percent of the cohort also received an IMiD-based mainte-

Table 1. Description of patients' characteristics and their association to early relapse.

\begin{tabular}{|c|c|c|c|c|c|}
\hline Variable & $\begin{array}{l}\text { No relapse within } \\
\begin{array}{c}18 \text { months } \\
n=2,131 \\
n(\%)\end{array}\end{array}$ & $\begin{array}{l}\text { Univariable analysis } \\
\text { Early relapse } \\
\begin{array}{c}\text { ( } \leq \mathbf{1 8} \text { months) } \\
n=496 \\
n(\%)\end{array}\end{array}$ & $\mathbf{P}$ & OR [CI 95\%] & malysis \\
\hline Sex & & & 0.711 & & \\
\hline Male & $1,198(58)$ & $286(58)$ & & & \\
\hline Female & $883(42)$ & 203(41) & & & \\
\hline Missing & 50 & 7 & & & \\
\hline Age & & & 0.422 & & \\
\hline$<60 y$ & $1,208(57)$ & $291(59)$ & & & \\
\hline$\geq 60 y$ & $923(43)$ & $205(41)$ & & & \\
\hline ISS & & & $<0.0001$ & & \\
\hline 1 & $633(36)$ & $88(22)$ & & 1.00 & \\
\hline 2 & 757 (43) & $181(45)$ & & 1.83 [1.18-2.82] & 0.007 \\
\hline 3 & 387 (22) & $136(33)$ & & $2.36[1.46-3.80]$ & $<0.001$ \\
\hline missing & 354 & 91 & & & \\
\hline Cytogenetic & & & $<0.0001$ & & \\
\hline standard risk & $1,560(85)$ & $300(67)$ & & 1.00 & \\
\hline high risk* & $268(15)$ & $145(33)$ & & 3.66 [2.51-5.34] & $<0.001$ \\
\hline missing & 303 & 51 & & & \\
\hline Treatment & & & 0.001 & & \\
\hline doublet & $755(35)$ & $215(43)$ & & $1.16[0.82-1.65]$ & 0.395 \\
\hline triplet & $1,376(65)$ & $281(57)$ & & 1.00 & \\
\hline Best response & & & $<0.0001$ & & \\
\hline VGPR/CR & $1,134(84)$ & $128(57)$ & & 0.24 [0.17-0.35] & $<0.001$ \\
\hline $\mathrm{SD} / \mathrm{RP}$ & $223(16)$ & $95(43)$ & & 1.00 & \\
\hline Missing & 774 & 273 & & & \\
\hline Maintenance & & & 0.362 & & \\
\hline No & $1,879(88)$ & $430(87)$ & & & \\
\hline Yes & $252(12)$ & $66(13)$ & & & \\
\hline
\end{tabular}

* high risk means presence of del(17p) and/or t(4;14). ISS: injury severity score ;VGPR : very good partial response; CR : complete response; SD: stable disease; RP: partial response. 

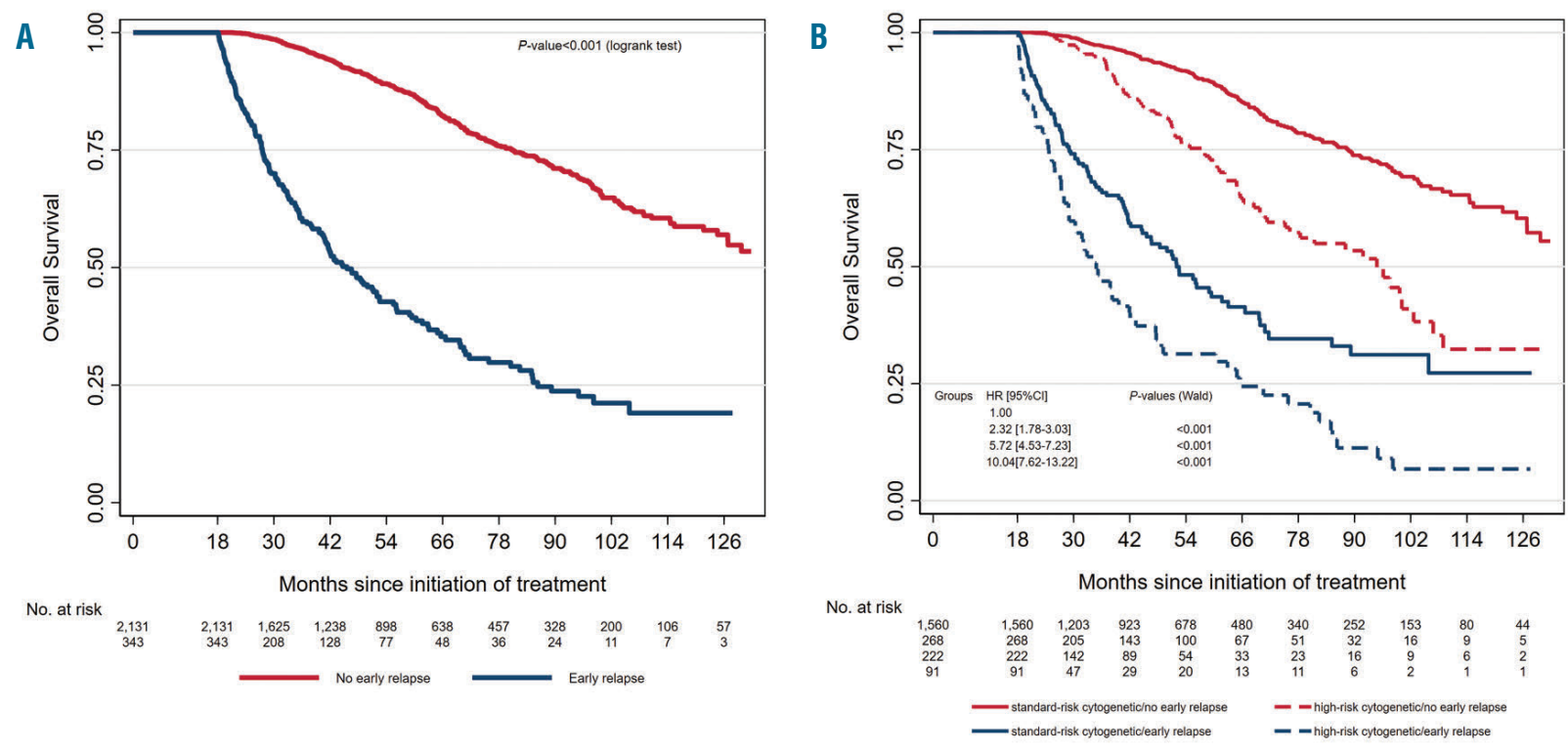

Figure 1. Kaplan-Meier overall survival curves for patients according to early or no early relapse. Landmark analysis at 18 months. (A) Patients with no early relapse (ER) $(n=2,131)$ versus patients with ER $(n=343)$. (B) Patients with no ER and standard-risk cytogenetic ( $n=1,560$, red line) versus patients with no ER and high-risk cytogenetic $(n=268$, red dashed line) versus patients with ER and standard-risk cytogenetic ( $n=222$, blue line) versus patients with ER and highrisk cytogenetic ( $n=91$, blue dashed line). High-risk cytogenetic was defined by the presence of $t(4 ; 14)$ and or del(17p) in more than $55 \%$ of plasma cells.

nance. Depth of response and progression were determined based on criteria defined by the International Myeloma Working Group. ${ }^{6}$ Relapse was considered early if it occurred within 18 months of starting initial therapy, which is equivalent to relapse within 12 months from ASCT. ${ }^{1,2}$ Survival analyses were performed using Landmark analysis at 18 months after initiation of treatment to reduce the guarantee time bias, reducing the cohort to 2,474 patients ( $\mathrm{n}=153$ excluded because followed $<18$ months, see the Online Supplementary Figure S1). The data was summarized by frequency and percentage for categorical variables and by median and range for continuous variables. The association of parameters with ER was tested with the $\chi^{2}$ or Fisher's exact test for univariable analyses, and logistic regression models for multivariable analyses. Survival rates were estimated by the Kaplan-Meier method. Univariable and multivariable analyses were performed using Logrank test and Cox proportional hazards model. Tests were two-sided and $P$-values $<0.05$ were considered significant. Statistical analyses were conducted using Stata ${ }^{\circledR}$, version 13.

Bone marrow samples were obtained at diagnosis and shipped overnight to a central laboratory. Upon receipt, plasma cells were isolated from bone marrow using CD138 ${ }^{+}$MAC-Sorting (Miltenyi Biotec, Paris, France). Post-sorting purity was checked as previously described and only samples with $\geq 70 \%$ plasma cells after sorting were kept for the cytogenetic analysis. Plasma cells were analyzed by fluorescence in situ hybridization for $\mathrm{t}(4 ; 14)(\mathrm{p} 16 ; \mathrm{q} 32)$ and $\operatorname{del}(17 \mathrm{p} 13)$ determination using specific probes from Abbott Molecular (Paris, France). Only deletions $17 \mathrm{p}$ present in $\geq 55 \%$ plasma cells were taken into account. ${ }^{7}$ For some patients, single-nucleotide polymorphism (SNP)-array was also performed (Affymetrix, Santa Clara, CA. USA) using the Cytoscan ${ }^{\circledR}$ array.

Amongst the 2,627 patients, 496 (18.9\%) experienced an ER. The remaining 2,131 patients $(81.1 \%)$ had either not relapsed at the time of analysis, or relapsed but after 18 months. A description of patients' characteristics and their association to ER is given in Table 1. According to both univariable and multivariable analyses, patients with an injury severity score (ISS) score of $2 / 3$, poor response to treatment and high-risk cytogenetics were more at risk of ER, whereas sex, age (including $>65$ years, data not shown) and presence of maintenance had no significant effect (Table 1). For this last point, it is noteworthy that only $12 \%$ of the patients were concerned, which is not surprising considering the relatively recent approval. A doublet-based treatment was more at risk of ER, but not independently (Table 1). The median follow up was 52.1 months (95\% confidence interval [CI]: 50.354.0) and the 5 years OS was $79.7 \%$ (range: $77.6-81.6$ ). According to univariable analysis, poor response to treatment $(P=0.004)$, age $\geq 60$ years at diagnosis $(P=0.006)$, doublet based treatment $(P<0.001)$, ISS 2 and 3 $(P<0.001)$, high-risk cytogenetics $(P<0.001)$ and ER $(P<0.001)$ (see Figure $1 \mathrm{~A}$ and Online Supplementary Table $S 1)$ were significantly associated with reduced OS, whereas sex and maintenance were not found associated to OS ( $P=0.098$ and $P=0.230$, respectively). According to multivariable analysis (see the Online Supplementary Table S2), the independent factors significantly associated with reduced OS were: (i) doublet-based treatment (hazard ratio [HR] 1.54 (range: 1.18-2.00), $P=0.0029$; (ii) ISS 2 (HR 1.72 (range: $1.24-2.38$ ), $P=0.001$ ) and ISS 3 (HR 1.89 (range: 1.33-2.68), $P=0.0003$ ); (iii) high-risk cytogenetics (HR 2.05 (range: 1.54-2.72), $P<0.0001$ ); and (iv) ER (HR 4.40 (range: $3.54-5.81$ ), $P<0.0001$, see Figure $1 \mathrm{~A}$ ). Figure $1 \mathrm{~B}$ represents the prognostic association of ER and cytogenetic risk.

Response to treatment has been a major prognostic factor in MM. It can be defined by at least three dimensions: rapidity, depth and duration. Fifteen years ago, Durie et al. showed that the underlying dominant predictor for OS is time to progression, ahead of depth of response to 
induction treatment. ${ }^{8}$ The Mayo Clinic has been the first to specifically describe the adverse prognostic impact of an ER after autologous transplant. ${ }^{1}$ Given the current availability of efficient salvage therapy, we wondered if an ER after intensive first line still negatively impacts survival. We demonstrate here that ER is still strongly associated with reduced survival after an intensive first line treatment, confirming three recent studies obtained with smaller cohorts, ${ }^{2,9,10}$ and that achieving at least a very good partial response (VGPR) is associated with lower risk of ER (adjusted overall response [OR] 0.24 [range: $0.17-0.35], P<0.001)$. Significant progresses has been recently made for response evaluation, and high sensitivity minimal residual disease may better predict ER. ${ }^{11}$ High-risk cytogenetics was also associated with ER (adjusted OR 3.66 [range: 2.51-5.34], $P<0.001$ ), as suggested by previous studies. For instance $t(4 ; 14)$ has been shown to induce a chemotherapy sensitive disease, characterized by a high OR rate, but patients achieved short PFS after transplantation. ${ }^{12}$ Very recently, revised-ISS has been shown to be predictive and prognostic for relapse within 24 months after ASCT, ${ }^{13}$ confirming that some initial characteristics are associated with higher probablity to experiment short PFS. But importantly, we observed that not all patients with ER were included in high-risk cytogenetic group, since $12.5 \%$ of standard-risk patients experienced an ER (vs. $25.4 \%$ of high-risk cytogenetic group, see Table 1). Of note, these patients died sooner than those belonging to the high-risk cytogenetics and no ER subgroup (Figure 1B). More recently, other cytogenetic factors have been described to negatively impact patient 'survival such as 1q gain and del(1p32), or positively such as trisomy $5 .{ }^{14}$ When this information was available $(840,820$ and 830 patients, respectively, see the Online Supplementary Figure S2 and Online Supplementary Table S3), we observed that 1q gain and del(1p32) were also associated with more likely ER whereas trisomy 5 was protective. Nevertheless, $14.7 \%$ of the patients without any of these four adverse prognostic factors (del(17p), $t(4 ; 14)$, 1q gain and $\operatorname{del}(1 \mathrm{p} 32))$ experienced ER after their first line treatment. A weakness of our study is that we did not include lactate dehydrogenase levels, these data being only available for patients diagnosed from 2015 onward. ${ }^{15}$ We also did not include the $t(14 ; 16)$, since in our experience it does not represent an independent prognostic parameter. Despite this, explanations are still unknown to define those patients. Our data could be the starting point to subsequently identify new genomic changes or immune dysfunctions associated with ER, in order to better identify this subgroup from diagnosis. In addition, a patient initially considered to be of favorable risk may also acquire high risk abnormalities such as deletion $17 p$ between treatment and relapse, underlying the need to also establish cytogenetic risk at relapse.

In conclusion, despite efficient post-relapse treatment, ER is strongly associated with reduced survival in both high- and standard-risk cytogenetic groups, and should be included in high-risk MM definition. Our data confirms that the prognosis established at diagnosis is a dynamic data which can evolve according to response to treatment, raising the question about the merit of current risk-adapted therapy and underlying the need to explore mechanisms of relapse. In addition, this study suggests that despite important progresses in salvage therapy, the first line of treatment must be the most efficient as possible.

Jill Corre, ${ }^{1,2^{*}}$ Lydia Montes, ${ }^{3 *}$ Elodie Martin, ${ }^{4}$ Aurore Perrot, ${ }^{5}$ Denis Caillot, ${ }^{6}$ Xavier Leleu, Karim Belhadj, ${ }^{8}$ Thierry Facon,
Cyrille Hulin, ${ }^{10}$ Mohamad Mohty, ${ }^{11}$ Jean Fontan, ${ }^{12}$ Margaret Macro, ${ }^{13}$ Sabine Brechignac, ${ }^{14}$ Arnaud Jaccard, ${ }^{15}$ Anne-Marie Stoppa, ${ }^{16}$ Frederique Orsini-Piocelle, ${ }^{17}$ Didier Adiko, ${ }^{18}$ Laurent Voillat, ${ }^{19}$ Faiza Keddar, ${ }^{20}$ Marly Barry, Helene Demarquette, ${ }^{22}$ Marie-Noelle Certain, ${ }^{23}$ Isabelle Plantier, ${ }^{24}$ Murielle Roussel, ${ }^{25}$ Benjamin Hébraud, ${ }^{25}$ Thomas Filleron, ${ }^{4}$ Michel Attal ${ }^{2,25}$ and Hervé Avet-Loiseau ${ }^{1,2}$ * $C$ and $L M$ contributed equally as co-first authors

${ }^{1}$ Unit for Genomics in Myeloma, Institut Universitaire du Cancer de Toulouse-Oncopole, University Hospital, Toulouse; ${ }^{2}$ Centre de Recherche en Cancérologie de Toulouse, Institut National de la Santé et de la Recherche Médicale U1037, Toulouse; ${ }^{3}$ Hematology Department, University Hospital, Amiens; ${ }^{B}$ Biostatistics Department, Institut Claudius Regaud, IUCT-O, Toulouse; ${ }^{5}$ Hematology Department, University Hospital, Nancy; ${ }^{6}$ Hematology Department, University Hospital, Dijon; ${ }^{7}$ Hematology Department, University Hospital, Poitiers; ${ }^{8}$ Hematology Department, University Hospital, Créteil; ${ }^{\circ}$ Hematology Department, University Hospital, Lille; ${ }^{10}$ Hematology Department, University Hospital, Bordeaux;

${ }^{11}$ Hematology Department, University Hospital, Paris; ${ }^{12}$ Hematology Department, University Hospital, Besancon; ${ }^{13}$ Hematology Department, University Hospital, Caen; ${ }^{14}$ Hematology Department, University Hospital, Bobigny; ${ }^{15}$ Hematology Department, University Hospital, Limoges; ${ }^{16}$ Hematology Department, Institut Paoli Calmettes, Marseille; ${ }^{17}$ Hematology Department, Department Hospital, Annecy; ${ }^{18}$ Hematology Department, Department Hospital, Libourne; ${ }^{19}$ Hematology Department, Department Hospital, Chalon-sur-Saône; ${ }^{20}$ Hematology Department, Department Hospital, Valenciennes;

${ }^{21}$ Hematology Department, Department Hospital, Boulogne-Sur-Mer; ${ }^{22}$ Hematology Department, Department Hospital, Dunkerque;

${ }^{23}$ Hematology Department, Department Hospital, Mâcon;

${ }^{34}$ Hematology Department, Department Hospital, Roubaix and

${ }^{25}$ Hematology Department, Institut Universitaire du Cancer

de Toulouse-Oncopole, University Hospital, Toulouse, France

Correspondence:JILL CORRE corre.jill@iuct-oncopole.fr

doi:10.3324/haematol.2019.236588

Acknowledgments: the authors would like to thank the Intergroupe Francophone du Myélome for providing patient samples, clinical data and financial support.

Funding: this work was supported by National Institutes of Health, National Cancer Institute grants PO1-155258 and P50-100707 (H.A.L.) and the Cancer Pharmacology of Toulouse and Region (CAPTOR) program. The Centre de Recherches en Cancérologie de Toulouse Team 13 is supported by the Fondation ARC (Association pour la Recherche sur la Cancer, grant PGA1*20160203788).

\section{References}

1. Kumar S, Mahmood ST, Lacy MQ, et al. Impact of early relapse after auto-SCT for multiple myeloma. Bone Marrow Transplant. 2008; 42(6):413-420

2. Jimenez-Zepeda VH, Reece DE, Trudel S, et al. Early relapse after single auto-SCT for multiple myeloma is a major predictor of survival in the era of novel agents. Bone Marrow Transplant. 2015;50(2):204208.

3. Fonseca R, Blood E, Rue M, et al. Clinical and biologic implications of recurrent genomic aberrations in myeloma. Blood. 2003; 101(11):4569-4575.

4. Gertz MA, Lacy MQ, Dispenzieri A, et al. Clinical implications of $\mathrm{t}(11 ; 14)(\mathrm{q} 13 ; \mathrm{q} 32), \mathrm{t}(4 ; 14)(\mathrm{p} 16.3 ; \mathrm{q} 32)$, and $-17 \mathrm{p} 13$ in myeloma patients treated with high-dose therapy. Blood. 2005;106(8):28372840 .

5. Harousseau JL, Attal M. How I treat first relapse of myeloma. Blood. 2017;130(8):963-973.

6. Rajkumar SV, Harousseau JL, Durie B, et al. Consensus recommendations for the uniform reporting of clinical trials: report of the International Myeloma Workshop Consensus Panel 1. Blood. 2011; 117(18):4691-4705.

7. Thakurta A, Ortiz M, Blecua P, al. High subclonal fraction of $17 \mathrm{p}$ deletion is associated with poor prognosis in multiple myeloma. 
Blood. 2019;133(11):1217-1221.

8. Durie BG, Jacobson J, Barlogie B, et al. Magnitude of response with myeloma frontline therapy does not predict outcome: importance of time to progression in southwest oncology group chemotherapy trials. J Clin Oncol. 2004:22(10):1857-1863.

9. Majithia N, Rajkumar SV, Lacy MQ, et al. Early relapse following initial therapy for multiple myeloma predicts poor outcomes in the era of novel agents. Leukemia. 2016;30(11):2208-2213.

10. Ong SY, de Mel S, Chen YX, et al. Early relapse post autologous transplant is a stronger predictor of survival compared with pretreatment patient factors in the novel agent era: analysis of the Singapore Multiple Myeloma Working Group. Bone Marrow Transplant 2016; 51(7):933-937.

11. Paiva B, Gutierrez NC, Rosinol L, et al. High-risk cytogenetics and persistent minimal residual disease by multiparameter flow cytometry predict unsustained complete response after autologous stem cell transplantation in multiple myeloma. Blood. 2012;119(3):687-691.

12. Jaksic W, Trudel $\mathrm{S}$, Chang $\mathrm{H}$, et al. Clinical outcomes in $\mathrm{t}(4 ; 14)$ multiple myeloma: a chemotherapy-sensitive disease characterized by rapid relapse and alkylating agent resistance. J Clin Oncol. 2005; 23(28):7069-7073.

13. Gopalakrishnan S, D'Souza A, Scott E, et al. Revised International Staging System is predictive and prognostic for early relapse $(<24$ months) after autologous transplantation for newly diagnosed multiple myeloma. Biol Blood Marrow Tr. 2019;25(4):683-688.

14. Perrot A, Lauwers-Cances V, Tournay E, et al. Development and validation of a cytogenetic prognostic index predicting survival in multiple myeloma. J Clin Oncol. 2019;37(19):1657-1665.

15. Palumbo A, Avet-Loiseau $\mathrm{H}$, Oliva $\mathrm{S}$, et al. Revised International Staging System for multiple myeloma: a report from International Myeloma Working Group. J Clin Oncol. 2015;33(26):2863-2869. 